\title{
A Game-Based, Physical Activity Coaching Application for Older Adults: Design Approach and User Experience in Daily Life
}

\author{
Monique Tabak, $\mathrm{PhD}{ }^{1,2}$ Frederiek de Vette, MSc, ${ }^{1}$ Hylke van Dijk, $\mathrm{PhD},{ }^{3}$ and Miriam Vollenbroek-Hutten, $\mathrm{PhD}^{1,4}$
}

\begin{abstract}
Objectives: In this article, we describe the design approach of a game-based, mobile coaching application for older adults (65-75 years) that aimed to stimulate physical activity (PA) in daily life. We evaluated older adults' experiences using this application compared with a standard coaching application in terms of engagement, motivation to be physically active, and in relation to the applied design features.

Development and Design: An iterative design approach was followed to develop the game-based coaching application, called WordFit. Step count data (FitBit) were used for the crossword-inspired game. The standard coaching application (the ActivityCoach) displayed FitBit data.

Materials and Methods: Participants were asked to use the ActivityCoach for 1 week first and then use WordFit for up to 3 weeks. Engagement was determined by logging actual use and motivation for PA by a daily in-app motivation question and step count data. Afterward, a semistructured interview was conducted.

Results: Twenty older adults participated (71.0 \pm 5.0 years). Of these, 16/20 actively used the ActivityCoach (13.1 \pm 10.0 days) and 8/20 used WordFit (14.8 \pm 9.5 days). For the latter, mean PA before WordFit use was $5852 \pm 3652$ steps/day, while mean PA using WordFit was $7236 \pm 3335$ steps/day. The interviews $(n=12)$ showed that step data and feedback on performance through the ActivityCoach/FitBit were prominent motivators for PA. WordFit was generally played as a stand-alone brain trainer. Cognitive challenge, in-game challenges, and challenges through emergent gameplay were important for engagement to use the game. Older adults did not play WordFit together.

Discussion: WordFit was not seen as a tool to help improve PA behavior. Enjoyment of the game concept could be improved by providing older adults with new and nontraditional gaming concepts before assessing game preferences. Follow-up studies should be conducted in a target group that is known for low adherence rates and that can benefit from the intervention provided.
\end{abstract}

Keywords: Physical activity, Older adults, Mobile gaming, Persuasive coaching, Engagement, Motivation

\section{Introduction}

D EPLOYING A MORE active lifestyle is considered important for older adults as physical activity (PA) is consistently associated with lower mortality rates, higher levels of cardiorespiratory and muscular fitness, higher levels of functional health, and better cognitive function. ${ }^{1}$ Despite these well-established benefits for health and well-being, the majority of older adults do not meet the recommended levels of $\mathrm{PA}^{2,3}$ and lead a sedentary lifestyle. ${ }^{4}$ Consequently, a growing number of interventions that promote daily PA have emerged, including technology-supported coaching applications for daily life.

Intervention studies regularly report high levels of dropout and limited use of the technology-supported applications. ${ }^{5-8}$ As stated by the WHO": "Medicine will not work if you do not take them," and assuming a dose-response relationship between the engagement of the user and the effects of the

\footnotetext{
${ }^{1}$ Biomedical Signals and Systems, Faculty of Electrical Engineering, Mathematics and Computer Science, University of Twente, Enschede, The Netherlands.

${ }^{2}$ eHealth Group, Roessingh Research and Development, Enschede, The Netherlands.

${ }^{3}$ Serious Gaming Group, NHL University of Applied Sciences, Leeuwarden, The Netherlands.

${ }^{4}$ Ziekenhuis Groep Twente, Almelo, The Netherlands.
} 
intervention, ${ }^{9}$ it is likely that engagement of behavioral interventions in general could positively affect treatment outcomes. ${ }^{10,11}$ Ongoing communication efforts (e.g., telephone contacts) that keep the patient engaged in interventions may be a simple and cost-effective strategy, ${ }^{12}$ which is also commonly applied in eHealth interventions. ${ }^{13,14} \mathrm{Gam}-$ ing technologies may offer a more efficient alternative to increasing engagement in using the technology, for example, through incentives. ${ }^{11}$ However, the use of game-based applications for behavior change in older adults remains scarce. $^{15,16}$

In gamification, engagement is supported by the integration of elements and mechanisms borrowed from games. Simple reward systems, such as points, appear to be a foundational feature in game-based applications. ${ }^{15,17}$ Following the self-determination theory, such reward systems that utilize extrinsic motivation could possibly inhibit intrinsic motivation and could therefore even counteract the establishment of sustained behavior change. ${ }^{18}$ To understand and analyze the interplay between engagement with the technology and behavior change, Yardley et al. ${ }^{19}$ make a distinction between the microlevel of moment-to-moment engagement with the technology-supported intervention and the macrolevel of engagement with the intervention goals for behavior change, which are closely connected. Detailed understanding of the features that influence engagement for both use of the technology and to motivate changes in behavior is important for development of the rapidly growing field of game-based coaching applications. However, the overlapping conclusion of recent articles is the incomplete description of game-based interventions and how game features are operationalized ${ }^{17,19}$ and that the influence of such game features on actual engagement is not yet established. ${ }^{20}$

In this article, we describe the design approach and evaluation of a game-based, mobile coaching application that aims to stimulate PA in daily life of older adults aged 65-75 years. The application combines behavior change techniques with gaming strategies to facilitate engagement with the intervention and target health behavior objectives simultaneously. We evaluated the older adults' experiences with this application compared with a standard (not game-based) coaching application in terms of engagement in using the application and motivation to be physically active. With our study, we aim to contribute to the knowledge base of tailoring game design to older adult's preferences and experiences in daily life. In the next paragraph, we first outline the design approach of the game-based application, from preferences to prototype. This is followed by evaluation of using the application in daily practice, as described in the Materials and Methods section and Results section.

\section{Development and design of a game-based coaching application}

Standard application: ActivityCoach. The ActivityCoach is a mobile phone application developed at Roessingh Research and Development, Enschede, The Netherlands, which aims to monitor and coach clinical and nonclinical populations into healthy active lifestyles. ${ }^{21}$ The activity behavior is monitored by the Fitbit Zip ${ }^{\circledR}$ step counter, which assesses the number of steps throughout the day. A smartphone is used for providing feedback to the user. Unlike the FitBit, users can see in real time the current number of steps in relation to their goal, distribution of steps today per hour and last week per day (Fig. 1). The PA goal was set manually by the health care professional through a web portal, preferably in consultation with the user during a regular consult. In addition, the therapist could see an overview of the participants and their activity behavior, as measured by the FitBit in the web portal. The ActivityCoach has proven to be effective for several target groups, ${ }^{2-24}$ but needs improvement in terms of use and adherence rates. ${ }^{6}$ As such, the game-based
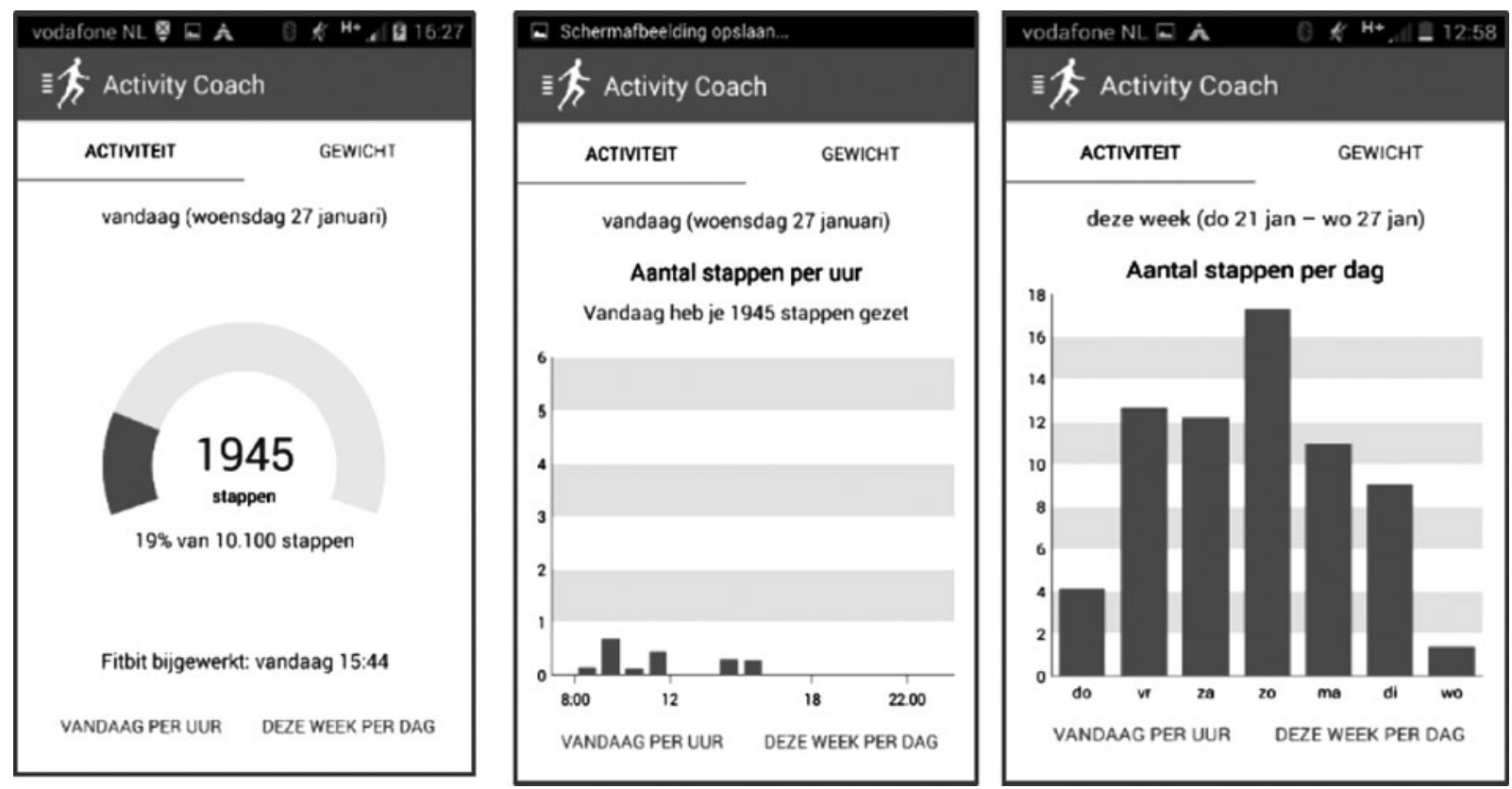

FIG. 1. Graphical user interface of the ActivityCoach. 
application will be added as a game layer to increase engagement with the application and reach the underlying health goals simultaneously.

Design approach of the game-based application. An iterative design approach has led to the development of a game-based coaching application (Fig. 2). First, we investigated the gaming preferences of older adults, as detailed in the next paragraph. These outcomes were incorporated in several lo-fi prototypes using a design thinking process. In addition, we matched specific game mechanics and features to the preferences of the older adult, as described in the study by de Vette et al. ${ }^{25}$ These findings on game preferences were implemented in the game-based application as follows.

Older adults' game preferences. To explore the current gaming behavior (i.e., gaming, gamification, and play; digital and nondigital) of older adults, including gaming preferences, gaming frequency, gaming context, and motivation to play games of older adults, we performed an exploratory survey among 159 adults aged between 50 and 91 years (mean $66.9 \pm 9.2$ years), of which $44 \%$ were males; $75 \%$ of the respondents played games (digitally (39\%), nondigitally (36\%), or both $(25 \%)$ ) and were invited to complete the whole questionnaire. In summary, we observed the following:

1. Devices: older adults prefer mobile devices and PC/ laptop to play digital games on (rather than consoles such as Xbox $^{\circledR}$ ).

2. Playing motivation: the most important reason to play a game is for relaxation (108 times), followed by to train the memory (59 times) and for challenge (42 times). A combination of these factors is appreciated.

3. Social interaction: players prefer playing with others (43\%), alone (39\%), and against others (18\%). A balance of competition and cooperation within games is appreciated.

4. Game type: older adults mostly enjoy puzzle-type games, often based on conventional games, for example, Yahtzee, Rummikub, and Scrabble.

In addition, we investigated which game characteristics should be applied to improve engagement for this specific target group. ${ }^{25}$ From this ongoing research, the following first results were taken into account in development of the game-based coaching application:

5. The older adult is achievement striving (rather than playing out of habit) and game content should satisfy their need for feedback on progress and successes. In addition, competition is highly valued, including challenging themselves. The older adult enjoys putting effort in a game to complete objectives.

6. The older adult enjoys variation in game content. Challenges should be made interesting by means of new content, leading to various outcomes. Games with a high esthetic value are appreciated.

7. Problem-solving and puzzle-themed games are found to be highly motivating. The target group is, however, open to modern games and new experiences.

8. Older adults are unspecific in their interest in interaction with others in or through digital games, while social contact is highly valued through traditional (board/card) games.

9. Older adults are easily demotivated by fast and stimulating gameplay and have a low tolerance for stressful and frustrating content. A trial-and-error approach is hardly ever taken and leads to loss of interest in the application or game. Frustration may also be avoided by enabling pausing and resuming the game at any point.

10. The older adult strongly dislikes any expression of violence.

Lo-fi prototypes. A design thinking process was used, in which several tangible prototypes were developed to create experiences with stakeholders. There were four alternating phases: (1) definition and analysis (identification of prerequisites and existent knowledge, values, and limitations), (2) ideation (development of concepts), (3) prototyping (creating the article and other lo-fi prototypes), and (4) experimenting (testing of mock-ups, concept, and values through focus groups with experts and play tests with older adults). The WordFit concept was developed toward a full prototype.

Game-based coaching application: WordFit. WordFit is a prototype game (Fig. 3) to engage older adults in use of the technology and thereby supports the older adult in deploying and maintaining an active lifestyle (Table 1). The abovementioned older adults' preferences (no. 1 to no. 10) were incorporated as follows: progression in the game depends on measured daily activity. The game is designed to be played on the smartphone or tablet, anywhere, anytime (no. 1), alone, or with others (no. 3 and no. 8). The game concept is a crossword puzzle inspired by conventional

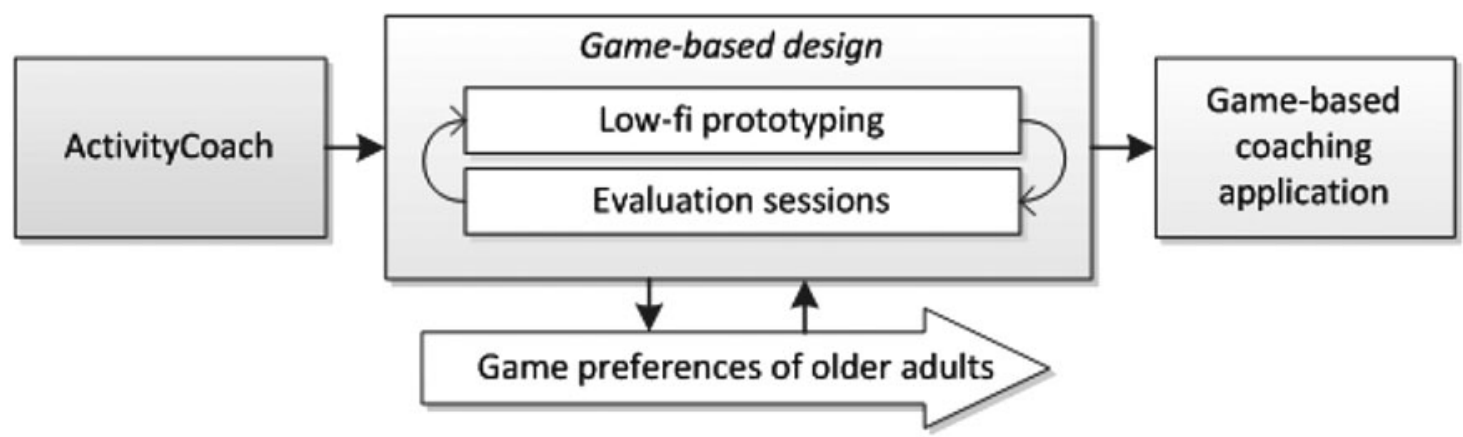

FIG. 2. Design approach for development of the game-based coaching application. 


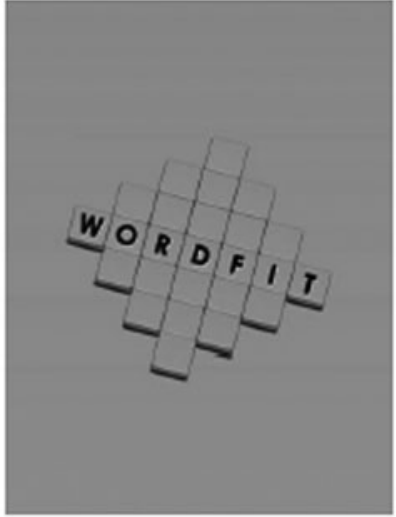

Splash screen

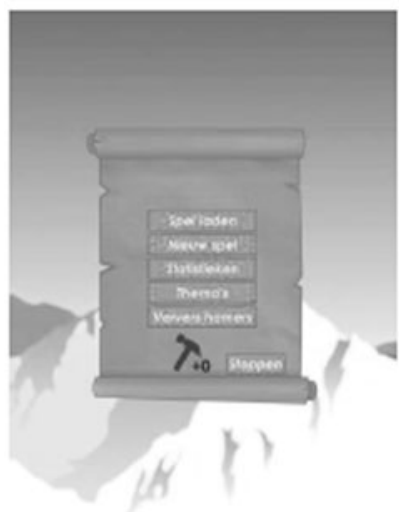

Main menu

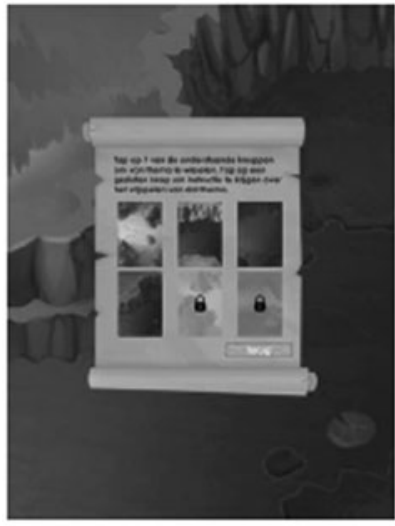

Unlock themes

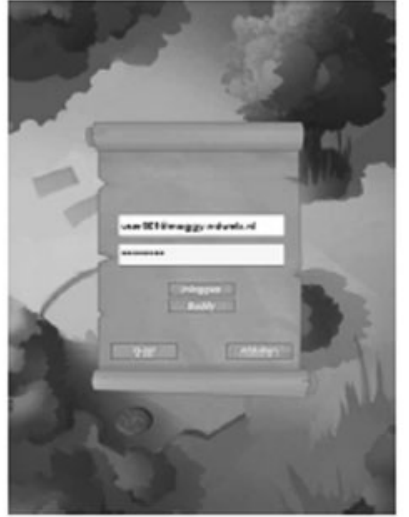

Log on screen

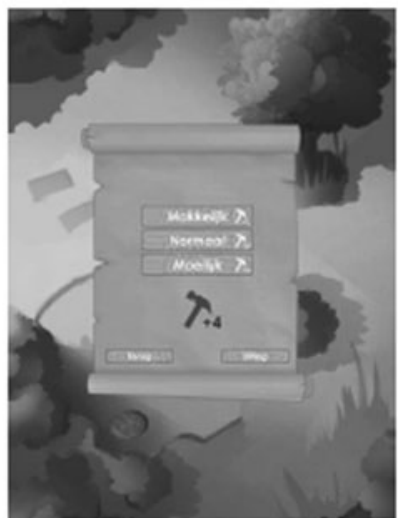

Choose difficulty level

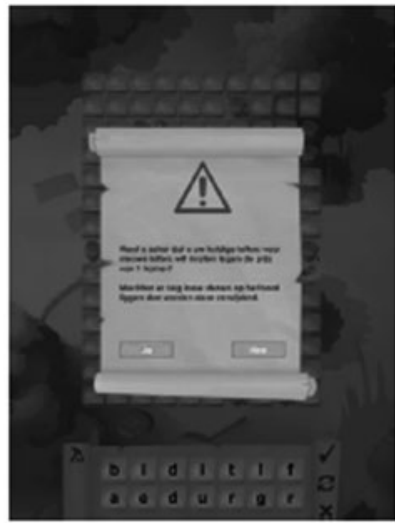

Change board for hammer

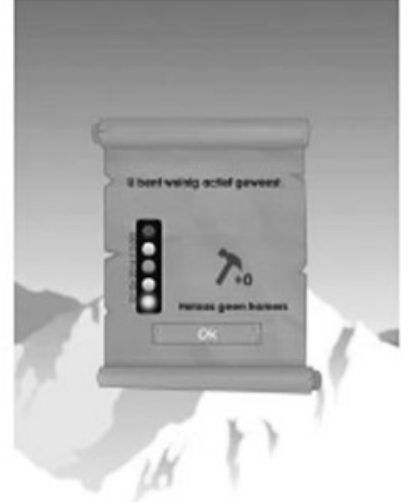

Activity feedback

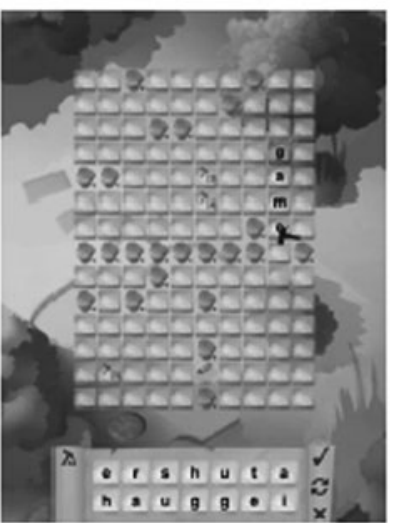

Board of crossword

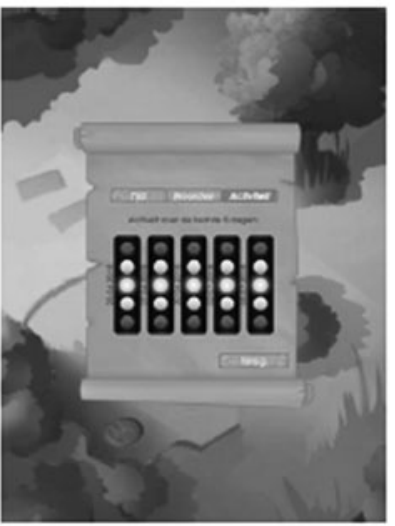

Scores: Activity overview screen

FIG. 3. Screenshots of WordFit. Different themes are shown (Forest lake, Snow mountain, and Rocky coast).

crossword puzzle games such as Scrabble (no. 2, no. 4, no. 7 , and no. 10). The main game mechanic is to place words from a starting point to an endpoint (no. 2 and no. 9). Rocks are placed on several tiles on the board, which need to be demolished by hammers to place words to reach the endpoint. The hammers can be earned when the step goal, measured by the sensor, is reached by the user (no. 5). In game, extra hammers can be collected by placing words on special tiles. Hammers are also used to rotate or swap one or more letters with new ones (no. 6). The user can open the game whenever he/she wants, although the hammers are only updated three times a day.

WordFit incorporates a combination of gaming strategies and behavior change techniques to facilitate engagement with the intervention and target health behavior objectives simultaneously, as discussed below and summarized in Table 2 .

Gaming strategies for engagement. The enjoyment of the gameplay itself is seen as the main strategy for engagement by gathering hammers from measured PA to 
Table 1. Characteristics of the Game-Based Application for Health: WordFit

Health topic(s) PA

Targeted age group(s)

Short description of game idea

Target player

Guiding knowledge or behavior change theory(ies), models, or conceptual framework(s)

Intended health behavior changes

Knowledge element(s) to be learned

Behavior change procedure(s) (taken from the Michie inventory) or therapeutic procedure(s) employed

Clinical or parental support needed?

Data shared with parent or clinician

Type of game

Game components

Player's game goal/objective

Rules

Game mechanic(s)

Procedures to generalize or transfer what is learned in the game to outside the game

Virtual environment

Setting

Game platform(s) needed to play the game

Sensors used

Estimated play time
Older adults (65-75 years)

WordFit is a game-based application connected to an underlying coaching application (ActivityCoach). WordFit aims to increase engagement of the older adult with the application, thereby supporting the older adult in deploying and maintaining an active lifestyle. The main game concept is a crossword puzzle that can be played on mobile devices. PA, as measured by a sensor, drives in-game mechanics.

Individual (optional guest or buddy player)

Self-monitoring of PA to create awareness, goal setting, and feedback on performance. Cognitive challenge, social play, enjoyment, achievement, and autonomy.

PA behavior, performance toward the personal goal.

Insight into PA behavior

Persuasion and incentivization within WordFit. The application was part of regular care where other additional procedures (e.g., education and training) were used to achieve health goals.

Yes, physical therapist

Yes, through web portal, showing PA

Puzzle, strategy, casual

Obtain as many hammers as possible to finish puzzles. Solve and create challenges. Improved activity behavior enhances rewards and creates and enables more satisfying gameplay.

Rocks are placed on several tiles on the board, which need to be demolished by hammers to place words to reach the end-point. The hammers can be earned when the step goal, measured by the sensor, is reached by the user. Themes and challenges can be reached in game. Creative emergent gameplay is stimulated. Elements enabling achievement are present through leaderboards, which can be shared with other users.

Gaming motivation, to obtain hammers and enable reaching in-game goals, is translated into real-world motivation to be physically active. Performance directly drives the game's progression. Specific strategies and implemented features are shown in Table 2.

WordFit aimed to create sustainable engagement with the technology, thereby creating a positive user experience in monitoring and adopting healthy behavior in daily practice.

PA is measured throughout the day with an activity sensor;

WordFit can be played on mobile devices in any environment.

Smartphone or tablet

FitBit Zip to measure PA (steps).

5-30 min/day

PA, physical activity.

proceed in the game. As we aimed to avoid explicit extrinsic rewards (e.g., through point systems), more unobtrusive reward systems are applied to the game. Positive rewarding is applied by offering more hammers when players are closer to their personal goal; the better the activity behavior, the better the award and more satisfying the gameplay.

A second important strategy is autonomy, a player can freely choose playing approaches, for example, through different difficulty levels or deciding to unlock certain challenges for changing the theme. This feeling of being in control is reinforced by the right of the user to add words to the library of the game. Furthermore, boards can be saved and reopened at any time. Autonomy is also reinforced by enabling emergent gameplay, playing a game outside its intended rules. For example, upon finishing a board, some game statistics are displayed such as time spent finishing this level and number of words used. Players are thereby triggered to invent their own gameplay and rules, which may lead to a more meaningful experience. 
Table 2. Overview of the Engagement Strategies InCORPorated in WordFit

\begin{tabular}{|c|c|c|}
\hline & Gamification & Behavior change \\
\hline Strategy & $\begin{array}{l}\text { Enjoyment } \\
\text { Autonomy } \\
\text { Emergent gameplay } \\
\text { Social play } \\
\text { Achievement }\end{array}$ & $\begin{array}{l}\text { Awareness of activity behavior } \\
\text { Goal setting } \\
\text { Feedback on performance } \\
\text { Positive reinforcement }\end{array}$ \\
\hline $\begin{array}{l}\text { Gamification } \\
\text { features }\end{array}$ & $\begin{array}{l}\text { Crossword puzzle } \\
\text { Unobtrusive rewarding (hammers) } \\
\text { Social accounts (buddy, guest) } \\
\text { Game statistics, personal leaderboard } \\
\text { Unique playing boards } \\
\text { Unlockable themes } \\
\text { Challenges (to unlock themes) } \\
\text { Difficulty levels } \\
\text { Expandable dictionary }\end{array}$ & $\begin{array}{l}\text { Traffic light metaphor represents PA } \\
\text { Review of past week's activity in statistics } \\
\text { Linking back to original ActivityCoach for detailed activity data } \\
\text { Goal achievement (optional: goal setting through a portal) } \\
\text { In-game feedback messages on activity performance }\end{array}$ \\
\hline
\end{tabular}

These in-game statistics may also be compared with others to invite for competition. This third strategy, social play, is supported by the possibility for peers or friends to $\log$ into one of two extra accounts implemented in the game. A buddy may log in with unique credentials and play WordFit under the same conditions as the main player, thus copying the activity level of the primary user. The rationale behind this is that the buddy can support and participate in the daily activity level of the primary user off-line. A guest account is available for free play with random activity levels in case a player does not want to share the activity data.

The game allows for achievement/skill development through the game statistics and a personal leader board, including time played to finish a game, words and hammers used, difficulty levels played, and activity of the past week. Three difficulty levels are implemented: the higher the level, the higher the number of rocks present on the board. To satisfy the need for variety in the game, each time the game is started, a new unique board is created. Second, optional achievements are created by challenging the user to complete a game within the set specifics. For example, a challenge can ask to finish a puzzle on the highest difficulty level within 60 seconds. These challenges also satisfy the need for variation as they are rewarded with new backgrounds for the application. Challenge may also emerge from social play and use of the buddy or guest accounts, stimulating engagement in both the game and the activity goals.

\section{Game-based coaching to motivate for PA}

Following principles of behavior change theories, the game-based coaching application provides self-monitoring of activity behavior to create awareness, facilitates goal setting, and provides feedback on performance.

To provide game-based feedback, the PA data from the ActivityCoach are used as input for the game. After logon, a traffic light appears every time the app is started by the player to provide insight into the activity behavior in the game (awareness). The player should be active according to his/ her personal goal (goal setting) that was set by the physical therapist through a web portal. Data are aggregated over predefined intervals, typically 00:00-12:00, 12:00-17:00, and 17:00-20:00, and compared with the prescribed goal activity for that interval.

PA could be too low ( $\geq 20 \%$ deviation below the goal), slightly too low ( $\geq 10 \%$ deviation below the goal), OK ( $<10 \%$ deviation), slightly too high $(\geq 10 \%$ deviation above the goal), or much too high ( $\geq 20 \%$ deviation above the goal). This is subsequently mapped to a five-point scale (-2 through 2). In game, the activity level throughout the day was visualized by means of a metaphor with safety margins - a traffic light $(0=$ green, $-1,1=$ orange, and -2 , $2=$ red). In addition, the traffic light is accompanied by a short message about the activity behavior (feedback on performance) and consequently how many hammers the user receives (positive reinforcement). These messages to support the older adult are friendly, short, generic, and without strong arguments. In addition, the player can see an overview of the measured activity levels of the past 5 days, in traffic lights. WordFit offers a quick link to the ActivityCoach from the game if more detailed information on the measured steps is desired.

\section{Materials and Methods}

\section{Study design}

The aim of the evaluation study was to evaluate the older adults' experiences of the game-based application compared with a standard coaching application in terms of engagement in using the application and motivation to be physically active. The study setting is in the daily life of the older adult, under supervision of the physical therapist at a distance.

\section{Participants}

Participants were eligible for inclusion if they fulfilled the following criteria: 65-75 years old upon inclusion and no health-related complaints that limit the performance of physical activities in daily life (able to comply with step goal) as determined by their physical therapist. Participants were recruited from the physical therapy practices PMI Rembrandt in Veenendaal, de Klomp in Enschede, and the physical therapy departments from the elderly care organization TriviumMeulenbeltZorg in Almelo, Hengelo, and Borne, The Netherlands. The study was approved by the 
University of Twente Ethics Committee and all participants provided their written informed consent.

\section{Protocol}

The total study duration was 4 weeks. In the first week, participants were asked to use the standard application, ActivityCoach, which connects with Fitbit. In the following weeks, participants were asked to use WordFit for as long as they wanted, with a maximum duration of 3 weeks. The daily step goal was set to a standard of 6000 steps/day, as determined by the physical therapists involved. The physical therapist could interact with the system through the web portal to set a personal goal that was agreed upon between the therapist and the participant as part of regular treatment. Additionally, therapists could personalize the goal to fit the capabilities of the individual participant if needed. The participant was free to choose if, when, and how often the applications were used.

\section{Measures}

To determine engagement, objective use data were automatically gathered for the FitBit, ActivityCoach, and WordFit and saved in log files, from the first to the last measurement day. A day of use was defined by the presence of step data and counts of at least 100 steps; otherwise, the day was not used for analyses. One day of use in the ActivityCoach was counted when the app has been opened. One day of use of WordFit was counted when at least one word has been placed on the board within the game.

The motivation to be active was measured by experience sampling on a daily level, by asking on a scale of 1 to 7 each day on the mobile device. In addition, the FitBit was used for registration of PA and expressed in steps/day.

Semistructured interviews after the study were conducted to acquire in-depth understanding of the user experience with regard to engagement with the technology, motivation to change activity behavior, and overall experience with both applications and the game features incorporated. Example questions included: Was playing WordFit engaging for you? Why (not)? What was your most important reason to be physically active? What are the strong and weak points of WordFit?

\section{Data analyses}

For engagement, the successive use days, mean (standard deviation [SD]) number of use days, and the amount of PA are presented for the FitBit, ActivityCoach, and WordFit. We stratify these outcomes for active ( $\geq 2$ days of use) and nonactive users ( $0-1$ day of use). Application use is graphically presented over the course of the study. For the motivation score and PA data, the mean (SD) is presented separately for the days before using WordFit and for the days WordFit use was started. As this was an exploratory study to investigate user experiences and not (clinical) effects, no power calculation was done beforehand and no statistical analyses are performed.

For the interviews, a mixed-analysis approach was used, in which we not only analyzed data qualitatively, following content analysis, ${ }^{26}$ but also quantified the data. The interviews were audio recorded and transcribed. The transcripts were read and reread and coded by F.d.V. and M.T. separately to identify particular features of the data set with regard to user experience and usability, engagement, and motivation. These codes were categorized per application (i.e., FitBit, ActivityCoach, and WordFit). Next, game strategies and features were identified and categorized per application. Each identified strategy or feature was categorized in one of the overarching themes corresponding to our research questions on engagement to use or motivation to be physically active (as mentioned in Table 2). Discrepancies were discussed until consensus was reached. The number of times unique participants mentioned the identified game strategies and features as either motivating or demotivating was counted.

\section{Results}

\section{Participants}

Twenty-one older adults were recruited, one person dropped out before the start of the study due to illness. Twenty older adults participated in the study (mean age $71.0 \pm 5.0$ years) (Table 3). Eight older adults dropped out before the end of the study (unknown reason [ $n=3]$, not compatible with lifestyle $[n=1]$, too much effort $[n=1]$, becoming overly active [ $n=1]$, illness $[n=1]$, and private event $[n=1])$, meaning that 12 older adults participated in the semistructured interview held after 4 weeks.

\section{Engagement in using applications}

The total sum of successive use of all participants was 466 days, with a mean successive use of $23.3 \pm 11.0$ days. Engagement in using the applications varied greatly: all participants used the FitBit and 16/20 participants used the standard ActivityCoach application, of which 7 participants also played the WordFit game. One participant played WordFit, but did not use the ActivityCoach, and 4/20 participants used the FitBit without the applications.

Mean use of active users $(n=16)$ for the ActivityCoach was $13.1 \pm 10.0$ days (range: $2-36$ ) and mean use of active users $(n=8)$ for WordFit was 14.8 \pm 9.5 days (range 4-32). Figure 4 shows the number of active users per week, per application. This figure also shows that participants did not follow the protocol saying that WordFit should be used from week 2 onward. The buddy and guest accounts were rarely used.

Table 3. Participant Characteristics $(N=20)$

Participant characteristics

Age $(n=19)$

Gender (male/female)

Stage of change $(n=18)$

Technology use $(n=18)$

$71.0 \pm 5.0$ years $(n=19)$ $10 / 10$

Precontemplation: $n=0$ Contemplation: $n=1$

Preparation: $n=4$

Action: $n=2$

Maintenance: $n=11$

Daily: $n=8$

Weekly: $n=0$

Monthly: $n=3$

Rarely: $n=3$

Never: $n=4$ 


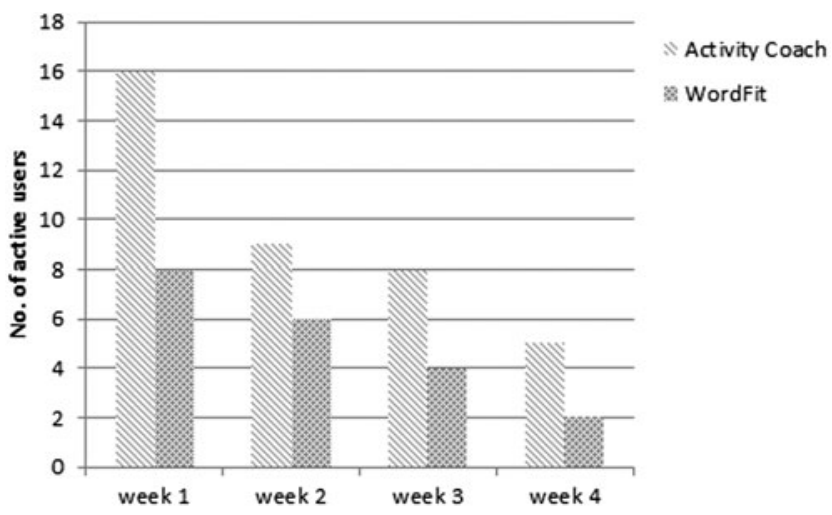

FIG. 4. No. of users per week, stratified per application.

\section{Motivation to be physically active}

Ten participants answered the daily motivation questions. The mean motivation to be physically active for the days before starting use of WordFit was rated $4.8 \pm 1.5(n=53)$ and $6.0 \pm 1.2$ for the days after starting use of WordFit $(n=140)$.

The overall mean daily PA level for the days before starting use of WordFit was $5235 \pm 3389$ steps $(n=17)$. For active WordFit users $(n=8)$, mean daily PA level for the days before starting use of WordFit was $5852 \pm 3652$ steps, while the mean steps/day for the days after starting use of WordFit was increased to $7236 \pm 3335$ steps. Six participants had measurements days before using WordFit and after using WordFit, of which five showed an increase in PA (range: 282-2353 steps) and one a decrease of 1700 steps.

\section{User experience}

Twelve users participated in the interview, of which six were very active WordFit users (1-3 weeks), two were active users (max. 1 week), and four were nonactive users.

Motivation to be physically active. The majority (9/12) of the interviewed participants found the FitBit motivating to be physically active. Reasons were noting the number of steps and comparing their activity behavior with previous days or with other persons, which motivated them to put slightly more effort in being active each day. Five participants wanted to continue using the FitBit after the end of the study and considered buying one.
One participant indicated that the number of steps does not represent the total daily activity very well. Six users were motivated to be physically active by the ActivityCoach because the ActivityCoach provided deeper insight into the daily activity behavior and in relation to the goal.

Only two participants indicated that they were motivated to be active because of the WordFit game: "Just because you are working on your physical activity in a pleasant way helps you to become more active," while others were clearly not motivated: "This [WordFit] did not activate me. That would not help me at all, a game to motivate me to become more physically active. That was already clear from the beginning." Although the game did not motivate most, they would recommend the game to others: "Yes, it could work for other people, it really depends whether you like the game itself.' Social contact was also seen as a motivator for PA, however, not as part of the applications, but in real life: "You talk about it with each other, about the number of steps you take every day, also when walking together. That's fun to compare.'

From the interviews, four game features were identified with regard to motivation to be physically active (Fig. 5). The connection between PA and the WordFit game, in general, was not clear to four users. The traffic light metaphor did not find a positive response among the participants. The traffic light was found to be unclear, incorrect, or insufficiently informative as a direct message on activity performance as well as a means to review past week's activity: "Information from the traffic lights alone was not enough." Some users also indicated that the in-game feedback was unclear or too harsh, resulting in annoyance: "[the system said] 'too little activity, go do something,' while I already walked 5800 steps and then I think: bugger off!' Linking back to the original ActivityCoach for checking detailed activity data was regularly used by five participants, while two other participants indicated that they did not use it as noting the steps were sufficient.

Engagement in using the applications. Six users enjoyed playing WordFit. In general, they liked to play a few times a day at home: "Yes, I liked it, just sit and play for a bit." An important factor for the experienced enjoyment was the puzzle/brain trainer aspect and creating and setting challenges. From the interviews, nine game features were identified regarding engagement to use the application (Fig. 6). The persons who did not like to play WordFit mainly disliked

\section{Motivation to be physically active}

\begin{tabular}{|c|c|c|c|c|c|c|c|c|c|c|c|}
\hline By Fitbit & + & + & + & + & + & + & + & + & + & $+1-$ & - \\
\hline By ActivityCoach & + & + & + & + & + & + & $+/-$ & $+/-$ & - & - & - \\
\hline By WordFit & + & + & - & - & - & - & - & - & - & & \\
\hline \multicolumn{12}{|l|}{ Features } \\
\hline Traffic light & - & - & - & - & - & - & - & - & & & \\
\hline In-game feedback message on PA & - & - & - & & & & & & & & \\
\hline In-game review at past week's activity (traffic lights) & - & - & - & - & - & & & & & & \\
\hline Linking back to ActivityCoach for detailed PA & + & + & + & + & + & - & - & - & & & \\
\hline
\end{tabular}

FIG. 5. Overview of positive (+), neutral (+/-), and negative (-) responses of the interviewed participants. 
Features

\begin{tabular}{|c|c|c|c|c|c|c|c|c|c|c|c|}
\hline \multirow[t]{2}{*}{ Cross-word puzzle / concept } & + & + & + & + & + & + & - & - & - & - & - \\
\hline & + & + & + & + & - & - & - & & & & \\
\hline Social accounts (buddy, guest) & - & - & - & - & - & - & - & & & & \\
\hline General emergent gameplay & + & + & + & + & + & + & & & & & \\
\hline Game statistics & + & + & $+/-$ & $+1-$ & - & & & & & & \\
\hline Themes & + & - & - & - & & & & & & & \\
\hline Challenges (to unlock themes) & + & + & + & + & & & & & & & \\
\hline Difficulty levels boards & + & + & $+/-$ & $+/-$ & $+1-$ & & & & & & \\
\hline Expandable dictionary & - & & & & & & & & & & \\
\hline
\end{tabular}

FIG. 6. Overview of positive (+) and negative (-) experiences of the gaming features to increase engagement in using the WordFit application.

the puzzle concept in general. Too little challenge or variation in the game was the other main reason for not enjoying the game: "It is too much like Scrabble." The unobtrusive rewarding by the hammers was positively valued by four participants. Five participants mentioned that the hammers were not needed for playing the puzzle and/or that it was too simple to obtain hammers in game (cheating) by choosing easy levels, thereby highlighting the multipurpose of the hammers: "It would have been nice if you could not earn hammers in the crossword puzzle. That it would be more a reward for the no. of steps you take" and "I started easy, then normal, then difficult, just to get the hammers." Some participants found it frustrating that the hammers were not saved for the next day.

Seven participants indicated that the guest and buddy accounts were not used (confirmed by the log data). Strikingly, when asked whether they would like to involve family of friends in the game, six participants responded positively.

Room for emergent gameplay, gameplay outside its intended rules, was generally found to be engaging. Participants set their own challenges, for example, gathering as many hammers as possible as well as creating their own cheating methods to receive more hammers. From the applied game elements, the challenges to unlock themes were valued most, while the expandable dictionary was valued least. People looked at the game statistics, but they were not necessarily engaged by the game statistics: "You see the statistics after playing, how long you played etc., and I looked whether I did better than the previous time." Unlocking themes was experienced to be fun, although not for getting a new theme, but for doing the corresponding challenge.

\section{Discussion}

WordFit was a first prototype game and research tool to explore the concept of a game-based application to support use of a behavior change intervention. Our study showed that WordFit was not seen as a tool or addition to help improve activity behavior. Instead, awareness of activity behavior by noting the number of steps and feedback on performance through the ActivityCoach and FitBit was the most prominent motivator for PA. The WordFit game was generally played as a stand-alone device for brain training. Emergent gameplay and challenge were the most prominent features for engagement with the technology. From our study, we observed the following factors that are beneficial for the experienced engagement and resulting success of the behavior change intervention.

\section{Design}

Six of the 20 participants actively engaged in using the WordFit application, which is comparable with the study by Spook et al., in which $28 \%$ of the people used the Balance It intervention. ${ }^{27}$ Important reasons in our study were the lack of perceived enjoyment of the puzzle concept and that the connection between PA and the game was not clear to the majority of users.

These findings are contradictory to our chosen design approach, which incorporates user preferences and lo-fi prototype evaluations. It seems that due to unfamiliarity with the envisioned technology, solely asking older adults about their game preferences was probably not sufficient. In the study by Bird et al., exposure to and participation in a serious game showed a dramatic increase in the older adults' perceptions of the usefulness of technology for improving health outcomes, including PA. ${ }^{28}$ The lo-fi prototyping in our study did not seem to provide sufficient interaction with the technology to really understand its implications, interactions, and components. As such, de Vette et al. ${ }^{25}$ provided older adults with a number of tablet games to play in daily life. Afterwards, the older adults' preferences for game content were mapped to the 5D framework, a classification for game content and a survey to assess user preferences for this content. This showed that older adults actually prefer new and nontraditional gaming concepts. ${ }^{25}$ When we use this framework to map the game content of WordFit and compare this with the older adults' user preferences, we see the discrepancy of the medium to low novelty in WordFit and the preferred high novelty of the older adult. In future design studies for new (gaming) technologies, we therefore suggest providing older adults with innovative gaming concepts before assessing their game preferences and letting them more actively experience the technologies during the design process. This may lead to new insights into their gaming preferences, which are valuable in designing an engaging gaming concept. 


\section{Target group}

The participants in this study were already highly motivated about increasing or maintaining their PA levels and positively influenced by gaining insight into their actual daily activity in the form of step counts during the study. As such, it is debatable whether the participants in our study needed the trigger and extra motivational support of the applications. This could explain that WordFit was mainly used as a brain trainer game. Engagement can be perceived as a dynamic process with four phases. ${ }^{19}$ WordFit was mainly designed to trigger engagement with the technology intervention (phase 1), thereby letting people experience the new behavior (phase 2). However, our participants were already actively involved in their PA behavior (corresponding to phase 3 ) and did not necessarily need the gamebased intervention. WordFit would only be of added value for our participants for reengagement with the technology in case of PA relapse (phase 4). Their already existing involvement in PA could explain why the standard coaching application showed higher engagement with the technology and the desired PA behavior, thereby removing the need for additional motivational support through a game-based application. To explore the added value of game-based coaching over traditional coaching, follow-up studies should be conducted with a focus on clinical effects in a target group that is known for low adherence rates in the use of PAsupporting applications and that can actually benefit from the intervention provided.

\section{Coaching}

Awareness of the activity behavior through the step data and feedback on performance (in relation to a goal) highly engaged the older adults to work on their activity behavior. The in-game feedback on PA was unclear and insufficiently informative and even counteracting user autonomy. This may have caused an early loss of interest in the game-based coaching app. In other studies, older adults emphasize the importance of continuous feedback on their performance from peers or instructors for engagement. ${ }^{29}$ Interestingly, although indicated in the development phase, the option for social play was not used at all. The findings also suggest that while social contact in conventional games is appreciated, older adults mostly prefer single-player digital games. The latter was also found in previous research on gaming preferences. ${ }^{30}$ Continuous feedback from the FitBit could have fulfilled the older adults' need for continuous feedback, making the gaming features more or less redundant for reaching engagement. A comparable result was found in the study by Muller et al., in which an interactive intervention to promote PA in diabetes type 2 showed lower engagement compared with the plain-text intervention. ${ }^{31}$ On the other hand, older adults in our study and in literature ${ }^{29}$ indicate that social contact would be an important motivator for PA outside the gaming context, in daily life. It would be worth investigating how social interaction can be incorporated in technology to help each other engage in PA.

\section{Challenge}

In our study, both in-game challenges, as challenges through emergent gameplay, were perceived as important for engagement to play WordFit. Challenge previously has been found to be the most popular motive to play a game. ${ }^{30}$ These challenges and emergent gameplay were intended to reinforce the feeling of autonomy and achievement in the player. This feeling could be improved by adding more variety in the game and improving some features (hammers and in-game feedback). Now, the older adults did not understand the connection between their PA behavior and the WordFit game, and the hammers were disputing their feeling of being in control and lack of user autonomy. Despite the low perceived motivation of the active WordFit users, visual inspection of their PA data showed that five of six even improved their PA behavior (not statistically tested). A similar result was found for the Balance It intervention, where exploratory analyses suggested that Balance It could contribute to changing PA behaviors when used as planned. ${ }^{27}$

\section{Context of use}

From previous research, we know the importance of embedding the technology in the overall treatment program, with the utmost important role for the health care professional. $^{32,33}$ In the study by Buimer et al., the behavior of professionals is shown to be an important incentive to use eHealth technology for rehabilitation as well as stimulation from fellow patients. ${ }^{34}$ In our study, the physical therapists were informed about the study, but only a few people from their practice participated, and thereby the study was not part of their regular routine. In addition, the technology should be part of daily life in general and fit the habits of the older adult. Our results show that the majority of older adults prefer to play at home, a few times a day, but mostly in the evening. This also accounted for the FitBit, which was mostly checked at the end of the afternoon or early evening. Engagement with the technology for behavior change is influenced by many factors, including diverse target group characteristics, context of use, and mode of delivery, ${ }^{19,35}$ and their inter-relations are complex. In future large-scale studies, clear communication to patients about the benefits of the technology, expectation management, and incorporation in the off-line (group) training program and in daily life would probably stimulate engagement.

\section{Strengths and limitations}

This study's strength is that it provides detailed insight into the implementation of design strategies and features for engagement. Future studies may benefit from the knowledge gained from this study regarding the game preferences of this group of older adults. The limitation of the study is that users did not strictly comply with the protocol to first use the ActivityCoach for 1 week without WordFit. However, as this study was exploratory, there was no aim to investigate differences between conditions.

\section{Lessons learnt}

From our research, we can distinguish several lessons learnt for designing a game-based coaching application for older adults, as shown in Table 4. 
TABLE 4. Lessons LeARNED

Older adults do not necessarily like traditional game concepts for game-based applications.

Let older adults experience innovative related technologies to elicit the correct requirements; solely asking about their preferences based on their prior experience is not sufficient.

In designing game-based coaching applications, the context of use should be carefully taken into account. For example, implementation strategies in daily routines (of older adults and health care professionals) should be designed alongside the application.

Choose an appropriate target group to evaluate: people who are not motivated to play in general will not be motivated by a game-based application. People who do not need or want to change PA behavior will not use the application as intended.

Feedback on PA should be highly informative and the role of the PA as input of the game should be extremely clear.

The user wants a feeling of autonomy. Sufficient and adequate challenge is key to sustaining engagement.

Social support could be of added value for engagement offline, for example, by exercising together.

\section{Acknowledgments}

The authors would like to thank the participants and the physical therapists from the care organizations PMI Rembrandt, De Kolk, and TriviumMeulenbeltZorg, for their active involvement in the study.

\section{Author Disclosure Statement}

No competing financial interests exist.

\section{Funding Information}

This research was part of the MAGGY (Mobile Activity Game for the ElderlY) project. Financial support was provided by The Netherlands Organization for Scientific Research, Creative Industry Program (project no. 314-99002).

\section{References}

1. WHO. Global Recommendations on Physical Activity for Health. Geneva, Switzerland: WHO Press; 2012.

2. Azagba S, Sharaf MF. Physical inactivity among older Canadian adults. J Phys Act Health 2014; 11:99-108.

3. Townsend N, Wickramasinghe K, Williams J, et al. Physical Activity Statistics 2015. London: British Heart Foundation; 2015.

4. Clemes SA, O'Connell SE, Edwardson CL. Office workers' objectively measured sedentary behavior and physical activity during and outside working hours. J Occup Environ Med 2014; 56:298-303.

5. Kohl LF, Crutzen R, de Vries NK. Online prevention aimed at lifestyle behaviors: A systematic review of reviews. J Med Internet Res 2013; 15:e146.

6. Tabak M, Brusse-Keizer M, van der Valk P, et al. A telehealth program for self-management of COPD exacerbations and promotion of an active lifestyle: A pilot randomized controlled trial. Int J Chron Obstruct Pulmon Dis 2014; 9:935-944.
7. Brouwer W, Kroeze W, Crutzen R, et al. Which intervention characteristics are related to more exposure to internetdelivered healthy lifestyle promotion interventions? A systematic review. J Med Internet Res 2011; 13:e2.

8. Davies CA, Spence JC, Vandelanotte C, et al. Meta-analysis of internet-delivered interventions to increase physical activity levels. Int J Behav Nutr Phys Act 2012; 9:52.

9. Vandelanotte C, Spathonis KM, Eakin EG, Owen N. Website-delivered physical activity interventions a review of the literature. Am J Prev Med 2007; 33:54-64.

10. Sabate E. Adherence to Long-Term Therapies. Evidence for Action. Geneva, Switzerland: World Health Organization; 2003.

11. Crutzen R, de Nooijer J, Brouwer W, et al. Strategies to facilitate exposure to internet-delivered health behavior change interventions aimed at adolescents or young adults: A systematic review. Health Educ Behav 2011; 38: 49-62.

12. Haynes RB, McKibbon KA, Kanani R. Systematic review of randomised trials of interventions to assist patients to follow prescriptions for medications. Lancet 1996; 348: 383-386.

13. van den Berg N, Schumann M, Kraft K, Hoffmann W. Telemedicine and telecare for older patients-A systematic review. Maturitas 2012; 73:94-114.

14. Dennison L, Morrison L, Lloyd S, et al. Does brief telephone support improve engagement with a web-based weight management intervention? Randomized controlled trial. J Med Internet Res 2014; 16:e95.

15. Tabak M, Dekker-van Weering $M$, van Dijk $H$, Vollenbroek-Hutten M. Promoting daily physical activity by means of mobile gaming: A review of the state of the art. Games Health J 2015; 4:460-469.

16. de Vette F, Tabak M, Dekker-van Weering M, VollenbroekHutten M. Engaging elderly people in telemedicine through gamification. JMIR Serious Games 2015; 3:e9.

17. Lewis ZH, Swartz MC, Lyons EJ. What's the point?: A review of reward systems implemented in gamification interventions. Games Health J 2016; 5:93-99.

18. Deci EL, Koestner R, Ryan RM. A meta-analytic review of experiments examining the effects of extrinsic rewards on intrinsic motivation. Psychol Bull 1999; 125:627-668; discussion 692-700.

19. Yardley L, Spring BJ, Riper H, et al. Understanding and promoting effective engagement with digital behavior change interventions. Am J Prev Med 2016; 51:833-842.

20. Brown M, O'Neill N, van Woerden $\mathrm{H}$, et al. Gamification and adherence to web-based mental health interventions: A systematic review. JMIR Ment Health 2016; 3:e39.

21. op den Akker H, Tabak M, Marin Perianu M, et al. Development and evaluation of a sensor-based system for remote monitoring and treatment of chronic diseasesThe Continuous Care \& Coaching Platform. In Proceedings of EHST 2012: The 6th International Symposium on eHealth Services and Technologies, Geneva, Switzerland; 2012.

22. Dekker-van Weering MG, Vollenbroek-Hutten MM, Hermens HJ. A pilot study-The potential value of an activitybased feedback system for treatment of individuals with chronic lower back pain. Disabil Rehabil 2015; 37:2250 2256.

23. Bruggeman-Everts FZ, Wolvers MDJ, van de Schoot R, et al. Effectiveness of two web-based interventions for chronic cancer-related fatigue compared to an active con- 
trol condition: Results of the "Fitter na kanker" randomized controlled trial. J Med Internet Res 2017; 19:e336.

24. Tabak M, op den Akker H, Hermens H. Motivational cues as real-time feedback for changing daily activity behavior of patients with COPD. Patient Educ Couns 2014; 94:372378.

25. de Vette A, Tabak M, Hermens HJ, Vollenbroek MMR. Mapping game preferences of older adults: A field study towards tailored gamified applications. In NordiCHI 2018. Oslo, Norway: ACM; 2018.

26. Elo $\mathrm{S}$, Kyngas $\mathrm{H}$. The qualitative content analysis process. J Adv Nurs 2008; 62:107-115.

27. Spook J, Paulussen T, Kok G, van Empelen P. Evaluation of a serious self-regulation game intervention for overweightrelated behaviors ("Balance It"): A pilot study. J Med Internet Res 2016; 18:e225.

28. Bird ML, Clark B, Millar J, et al. Exposure to "Exergames" increases older adults' perception of the usefulness of technology for improving health and physical activity: A pilot study. JMIR Serious Games 2015; 3:e8.

29. Kappen D, Nacke LE, Gerling KM, Tsotsos LE. Design strategies for gamified physical activity applications for older adults. In 2016 49th Hawaii International Conference on System Sciences. IEEE Computer Society; 2016, pp. 1309-1318. DOI: 10.1109/HICSS.2016.166.

30. De Schutter B. Never too old to play: The appeal of digital games to an older audience. Games Cult 2011; 6:155-170.

31. Muller I, Rowsell A, Stuart B, et al. Effects on engagement and health literacy outcomes of web-based materials promoting physical activity in people with diabetes: An
International Randomized Trial. J Med Internet Res 2017; 19:e21.

32. Mohr DC, Cuijpers P, Lehman K. Supportive accountability: A model for providing human support to enhance adherence to eHealth interventions. J Med Internet Res 2011; 13: e30.

33. Vermeire E, Hearnshaw H, Van Royen P, Denekens J. Patient adherence to treatment: Three decades of research. A comprehensive review. J Clin Pharm Ther 2001; 26: 331-342.

34. Buimer HP, Tabak M, van Velsen L, et al. Exploring determinants of patient adherence to a Portal-Supported Oncology Rehabilitation Program: Interview and data log analyses. JMIR Rehabil Assist Technol 2017; 4:e12.

35. Perski O, Blandford A, West R, Michie S. Conceptualising engagement with digital behaviour change interventions: A systematic review using principles from critical interpretive synthesis. Transl Behav Med 2017; 7:254-267.

Address correspondence to: Monique Tabak, PhD Biomedical Signals and Systems Faculty of Electrical Engineering, Mathematics and Computer Science University of Twente PO Box 217

7500 AE Enschede The Netherlands

E-mail:m.tabak@utwente.nl 